\title{
Early Sensitivity Training for Parents of Preterm Infants: Impact on the Developing Brain
}

\author{
JEANNETTE MILGROM, CAROL NEWNHAM, PETER J. ANDERSON, LEX W. DOYLE, ALAN W. GEMMILL, KATHERINE LEE,
} ROD W. HUNT, MERILYN BEAR, AND TERRIE INDER

\begin{abstract}
Department of Clinical \& Health Psychology [J.M., C.N., A.W.G.], Austin Health, Melbourne, Victoria 3081, Australia; Department of Psychology [J.M., P.J.A.], University of Melbourne, Victoria 3010, Australia; Departments of Obstetrics and Gynaecology [L.W.D.], and Paediatrics [L.W.D.], University of Melbourne, Victoria 3052, Australia; The Royal Women's Hospital [L.W.D., M.B.], Melbourne, Victoria 3052, Australia; Clinical Epidemiology and Biostatistics Unit [K.L.], Murdoch Childrens Research Institute [P.J.A., L.W.D., R.W.H., M.B.], Melbourne, Victoria 3052, Australia; Department of Neonatal Medicine [R.W.H.], The Royal Children's Hospital, Melbourne, Victoria 3052, Australia; and Department of Pediatrics, Neurology, and Radiology [T.I.], St. Louis Children's Hospital, Washington University, St. Louis, Missouri 63110
\end{abstract}

\begin{abstract}
After birth, preterm infants face a stressful environment, which may negatively impact early brain development and subsequent neurobehavioral outcomes. This randomized controlled trial involving 45 women with infants $<30$-wk gestation, assessed the effectiveness of training parents in reducing stressful experiences. Intervention consisted of 10 sessions in the Neonatal Intensive Care Unit (NICU). Postintervention, at term-equivalent (40-wk postmenstrual age), magnetic resonance imaging (MRI) was performed to evaluate brain structure and development. Quantitative volumetric techniques were used to estimate overall and regional brain volumes for different tissue types including CSF, CGM, DNGM, UWM, and MWM. DTI was used to evaluate the integrity and maturation of white matter by ADC and FA. Maturation and connectivity of white matter, characterized by diffusion MR measures of ADC and FA, were significantly enhanced in the intervention group, who displayed greater restriction in ADC and increase in FA. There were no significant effects on either brain volumes or on short-term medical outcomes. Thus, sensitivity training for parents in the NICU is associated with improved cerebral white matter micro-structural development in preterm infants. (Pediatr Res 67: 330-335, 2010)
\end{abstract}

$\mathrm{P}$ reterm infants are at an increased risk of persistent difficulties across most functional domains, evidenced by poorer academic achievement, well being, and productivity (1). These difficulties are associated with brain injury and with structural alterations that are commonly reported in preterm cohorts, from the neonatal period until later childhood and adolescence $(2,3)$. For example, white matter abnormalities are associated with cognitive and motor development (4) and reductions in brain volumes are related to some specific cognitive deficits (5-8).

However, neither these structural brain alterations nor the medical complications common in this population fully explain the variation in long-term neurobehavioral development $(9,10)$, and stressors in the child-rearing environment may also affect outcome $(11,12)$. Environmental sources of stress for preterm infants include exposure to the noisy, light-filled,

Received May 1, 2009; accepted November 7, 2009.

Correspondence: Jeannette Milgrom, Ph.D., Parent-Infant Research Institute. Department of Clinical \& Health Psychology, Austin Health, Melbourne, Victoria 3081, Australia; e-mail: jeannette.milgrom@austin.org.au

Supported by a grant from the Financial Markets Foundation for Children. busy environment of the Neonatal Intensive Care Unit (NICU), painful but necessary medical procedures and a paucity of parental contact. Animal and human studies confirm that deprivation of maternal contact imposes stress on neonates $(13,14)$. Modification of infants' physical and emotional environment while in NICU thus has the potential to reduce stress. Two environmental interventions aimed at doing this have shown promising results. The best known is the NIDCAP $(15,16)$ involving delivery of care without overwhelming infants' coping abilities and the scheduling of protected and supported rest periods for infants. In NIDCAP care, before and after necessary medical or nursing procedures, infants' behaviors are systematically recorded in terms of responses that are conceptualized as stress (e.g. skin coloring) or regulatory (e.g. grasping). Based on these observations of regulatory thresholds and strengths, the timing and implementation of all procedures are planned to reduce stress, together with provision of well-supported relaxation periods. Nurses and parents are provided with weekly feedback on infant responses. Benefits associated with NIDCAP care include earlier age at discharge, fewer days on oxygen, more regulated autonomic and motor systems, better orientation, tone, movement, posture, and fewer infants with bronchopulmonary dysplasia and intraventricular hemorrhage (16). Als et al. (11) followed up 30 low-risk preterm infants who had received NIDCAP care and also found enhanced neurobehavioral scores, more mature white matter at 2-wk corrected age (CA), and behavioral advantages at 9 mo of age.

In a less well-known longitudinal randomized trial Rauh et al. (17) and Achenbach et al. (18) assessed the effectiveness of the MITP Program $(n=91)$. The MITP also aims to reduce stress but in contrast to the NIDCAP, the mother is trained to facilitate the intervention. At $9 \mathrm{y}$ of age, IQ-equivalent scores

\footnotetext{
Abbreviations: ADC, apparent diffusion coefficient; CGM, cortical gray matter; CSF, cerebrospinal fluid; DNGM, deep nuclear gray matter; DTI, diffusion tensor imaging; FA, fractional anisotropy; MITP, mother-infant transaction program; MWM, myelinated white matter; NIDCAP, Newborn Individualized Developmental Care and Assessment Program; PLIC, posterior limb of the internal capsule; ROI, region of interest; UWM, un-myelinated white matter
} 
in the MITP intervention group were on average 10.6 points higher than the control preterm group. By using a modified version of the MITP ("PremieStart"), we found enhanced mother and infant behaviors as early as 6 to 12 mo of age due to the intervention (12).

We hypothesized that this intervention would buffer preterm infants from the harmful effects of acute and chronic stress, which in turn would result in enhanced brain development. The primary aim of this study was to investigate if this intervention was associated with improved brain development measured by magnetic resonance imaging (MRI) at termequivalent age. A secondary aim was to assess some possible short-term medical benefits.

\section{METHODS}

Design and sample. The study was a parallel, two-group controlled comparison of the "PremieStart" parent sensitivity training program, versus standard care (Trial Registration Number: NCT00883974), which was approved by the human research ethics committees of the Royal Women's Hospital and the Mercy Hospital for Women, both located in Melbourne, Australia. Between April 2004 and September 2005, women who delivered at $<30$-wk gestation at the NICUs of both hospitals were enrolled when infants were at 30 - to 32 -wk postmenstrual age. Recruitment operated $1 \mathrm{~d} / \mathrm{wk}$ on average, resulting in $81(20 \%)$ infants born at $<30$-wk gestational age (GA) being assessed for eligibility (Fig. 1). Demographic data were collected at enrollment. Educational booklets were given to all women during a short session explaining key components in the care of preterm infants. Excluded from enrollment were women having i) insufficient spoken and written English, ii) triplets or higher multiples, iii) infants with congenital abnormalities, or iv) homes $>100 \mathrm{~km}$ from Melbourne. Women were allocated to treatment conditions in a 1:1 ratio. Treatment allocation could not be masked from participants beyond the point of allocation. A computer-generated variable-length permuted blocks randomized allocation sequence was prepared by an independent person. Allocation concealment was ensured by a centralized system of sequentially numbered, sealed, opaque envelopes, which was overseen by a hospital administrator. After informed, written consent was obtained, and after all baseline data were recorded, women were allocated by assignment of the next envelope in the sequence.

Treatment conditions. The PremieStart parent sensitivity training program was delivered while infants were in NICU (nine sessions) with a homebooster session. Therapists (psychologists) worked with parents following a manualized protocol (12). Targets of intervention included training parents to recognize signs of infant stress, "shut-down" mechanisms, alert-available behavior, quality of motor behaviors, facial expressions, posture/muscle tone; principles of graded stimulation; how to optimize interactions and avoid
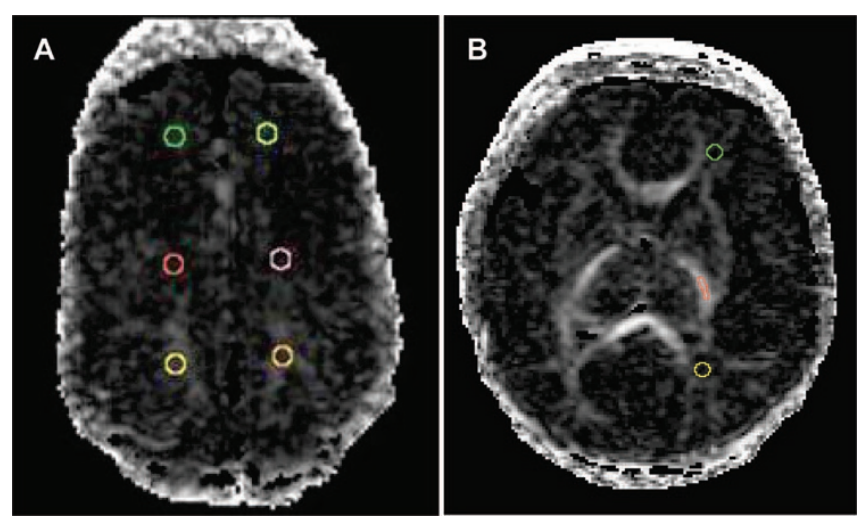

Figure 1. ROIs for diffusion tensor MRI in the superior and inferior axial slices. $A$. Image at the level just above the superior margins of the lateral ventricles. ROIs (marked on both hemispheres) in frontal, central (sensorimotor), and occipital white matter. $B$. Image at the level of the basal ganglia and posterior limbs of the internal capsule (PLICs). ROIs (marked only on one hemisphere) in frontal, central (middle third of PLIC), and occipital white matter. overwhelming infants; touch, movement and massage; "kangaroo care" (nesting infants skin-to-skin against their mother); vocal, visual and multi-sensory stimulation; normalizing parental feelings; challenging dysfunctional thinking, and diary keeping. A psychologist provided structured training to each intervention group mother in hospital, twice weekly for $2 \mathrm{wk}$, then weekly until discharge. The intervention included all the content of the original MITP intervention plus three new components: 1) massage 2) kangaroo care, and 3 ) a bath session to help mothers consolidate all they had learned about infant stress reactions in a standardized way. This increased content was possible because the intervention started earlier than the MITP and content was introduced and re-visited over the 6 to $8 \mathrm{wk}$ of the intervention (compared with one in-hospital week in the MITP). In addition, the content of the home visits (consolidation, mutual enjoyment through play, temperamental patterns and review, and termination) was collapsed into the final hospital sessions plus a single home visit at 4-wk postdischarge. This study also differed from the original MITP with regards to the inclusion of twins. Psychologists had only limited, administrative contact with hospital staff and discussed neither the content nor the progress of the intervention with them.

Standard procedures for the care of preterm infants occurred at both NICUs. At the time of this work, neither NIDCAP care, nor any specific interventions to decrease stress were being used. However, infants were handled minimally by staff and given "quiet time" when possible. Sucrose was given sublingually before painful procedures and in the first 2 wk after birth, approximately $1 / 4$ premature infants received morphine for analgesia.

Magnetic resonance imaging. Infants were scanned at term-equivalent age (38-42-wk postmenstrual age) by radiographers blinded to allocation, using a 1.5 Tesla General Electric Signa MRI scanner (Milwaukee, WI). To minimize motion artifact, infants were fed and swaddled, placed in a vacuum fixation beanbag, outfitted with earphones and scanned while sleeping. No sedation was administered.

T1- and T2-weighted images were acquired as per our previously reported protocol (4). MRI measurements were undertaken on Sun Microsystems workstations (Palo Alto, CA). Volumes of CSF, CGM, DNGM, MWM, and UWM estimated according to previously described criteria (19-21). For regional analyses, the brain was parcellated into 16 regions as previously described (8) with measurements for each parcel including total tissue and CSF volumes. For each voxel, three eigenvalues and eigenvectors of the diffusion tensor, and the ADC were calculated. Apparent diffusion was measured according to the Stejskal and Tanner equation (22) with the diffusion tensor elements estimated for each voxel by nonlinear regression. DTI analysis was performed off-line using XPhase image analysis software (SE Maier, Boston). ADC, FA, axial $\left(\lambda_{1}\right)$, and radial $\left(\left[\lambda_{2}+\lambda_{3}\right] / 2\right)$ diffusivity were calculated in 12 manually selected ROI in the brain located on two axial slices. Figure 1 illustrates the placement of ROIs on both slices from an infant enrolled in this study. The superior axial slice was taken at a level just above the lateral ventricles and ROIs were located in bilateral frontal, sensorimotor, and occipital white matter regions. The inferior slice was taken at a level through the DNGM and PLIC and ROIs were located in the bilateral middle third of the PLICs, and the frontal and occipital white matter regions.

Short-term perinatal and medical outcomes. These included data on intraventricular hemorrhage, days in hospital, postmenstrual age at discharge, sepsis, patent ductus arteriosus, indomethacin administration, hours of ventilation, and hours of $\mathrm{O}_{2}$, as well as postmenstrual age and body weight at MRI scan. Data on these secondary outcomes were collected blindly from hospital discharge summaries. In addition, weekly numbers of maternal visits to hospitalized infants, numbers of maternally administered bottle-feeds and numbers of maternal kangaroo-care periods were recorded in structured diaries that had been given to all parents.

Statistical analysis. A priori, power calculations based on detecting a $15 \%$ difference on MRI brain volume, with $80 \%$ power at $\alpha=0.05$, yielded $n=$ 27 per group. All analyses followed intention-to-treat principles (23). Baseline characteristics and perinatal data are presented for each group as proportions, means and standard deviations, or medians and interquartile ranges (25th to 75 th percentiles) as appropriate. Significance tests $\left(\chi^{2}\right.$ and MannWhitney $U$ tests) were carried out on several perinatal variables as these short-term health outcomes were also of interest.

Crude means and standard deviations within the two treatment conditions are presented for all MRI data. Comparisons of CSF, CGM, DNGM, MWM, and UWM volumes were made using separate linear regression models. Analyses are unadjusted for baseline characteristics in keeping with the randomized nature of treatment allocation (24). However, because brain size is related to age and also potentially to the intervention, we adjusted for postmenstrual age on the day of MRI scan as a sensitivity analysis. Results were unaffected and only unadjusted results are reported.

Differences in parcellated volumes and DTI parameters (ADC, FA, and axial and radial diffusivity) between the treatment groups were assessed using mixed 


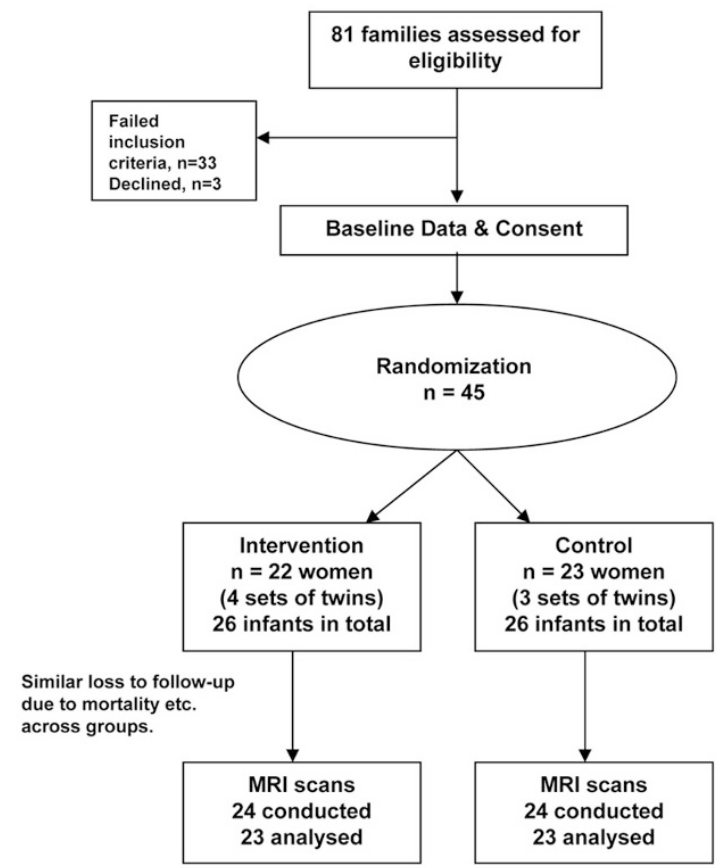

Figure 2. Participant recruitment and attrition.

Table 1. Participant characteristics

\begin{tabular}{lcc}
\hline & $\begin{array}{c}\text { Control } \\
\text { group }\end{array}$ & $\begin{array}{c}\text { Intervention } \\
\text { group }\end{array}$ \\
\hline Mothers & $n=23$ & $n=22$ \\
Mean age in years (SD) & $31.4(3.7)$ & $32.2(3.8)$ \\
English spoken at home, $n(\%)$ & $21(91)$ & $22(100)$ \\
Completed 12 years of school, $n(\%)$ & $20(87)$ & $19(86)$ \\
University degree, $n(\%)$ & $12(52)$ & $13(59)$ \\
Infants & $n=26$ & $n=26$ \\
Singletons, $n(\%)$ & $20(77)$ & $18(69)$ \\
Female, $n(\%)$ & $12(46)$ & $15(58)$ \\
Mean birth weight in grams (SD) & $999(307)$ & $981(241)$ \\
Mean gestational age in weeks (SD) & $27.7(1.6)$ & $27.5(1.9)$ \\
Antenatal maternal corticosteroids, $n(\%)$ & $18(69)$ & $19(73)$ \\
Breech presentations, $n(\%)$ & $4(15.4)$ & $8(30.8)$ \\
Unplanned caesarean extractions, $n(\%)$ & $3(11.5)$ & $8(30.8)$ \\
Resuscitations at birth, $n(\%)$ & $22(85)$ & $21(81)$ \\
Median APGAR scores (IQR)* & & \\
1 min & $5.5(3-7)$ & $5.5(4-7)$ \\
5 min & $8(7-9)$ & $8(7.2-9)$ \\
Died before reaching term-age, $n(\%)$ & $1(0.02)$ & $1(0.02)$ \\
Could not attend MRI scan, $n(\%)$ & $1(0.02)$ & $1(0.02)$ \\
MRI scan unusable, $n(\%)$ & $1(0.02)$ & $1(0.02)$ \\
\hline
\end{tabular}

* IQR, interquartile range.

models with all regions included in the same model as used previously (3). Correlations among repeated within-subject observations (multiple regions within the same infant) were modeled by including a random intercept for each infant. Heteroscedasicity between brain regions is modeled by allowing both the within and between subject variability to vary by region. Region comparisons were then made combining the results across hemisphere after checking for potential interactions. Again, the unadjusted results are presented because there was little difference after adjustment for postmenstrual age.

All analyses used the simplifying assumption that observations were independent. Sensitivity analyses using generalized estimating equations (GEEs) to allow for the correlation between twins in the linear regression models, and combining twin data into a single observation using the mean for each sibling pair made very little difference to results (not presented). Analyses were performed using Stata 10.1, SPSS 16, and SAS version 9.1.
Table 2. Mean (SD) cerebral tissue volumes and regional brain volumes by treatment group

\begin{tabular}{|c|c|c|}
\hline & $\begin{array}{l}\text { Control } \\
(n=23)\end{array}$ & $\begin{array}{l}\text { Intervention } \\
(n=23)\end{array}$ \\
\hline \multicolumn{3}{|l|}{ Tissue volumes (all regions combined) } \\
\hline Cortical grey matter & $189.9(29.6)$ & $187.1(32.9)$ \\
\hline CSF & $42.2(16.3)$ & $47.5(19.6)$ \\
\hline MWM & $6.9(3.1)$ & $7.0(3.2)$ \\
\hline UMWM & $216.7(29.5)$ & $214.2(30.7)$ \\
\hline Deep nuclear grey matter & $20.3(5.6)$ & $22.2(6.2)$ \\
\hline \multicolumn{3}{|l|}{$\begin{array}{l}\text { Regional volumes (all tissue types } \\
\text { combined) }\end{array}$} \\
\hline Orbitofrontal—right & $9.2(2.3)$ & $8.2(2.0)$ \\
\hline Orbitofrontal-left & $9.3(3.0)$ & $8.0(2.5)$ \\
\hline Dorsal prefrontal—right & $24.3(3.7)$ & $23.2(5.1)$ \\
\hline Dorsal prefrontal-left & $21.6(2.9)$ & $21.0(4.3)$ \\
\hline Premotor-right & $24.3(3.8)$ & $25.0(4.0)$ \\
\hline Premotor-left & $21.9(3.2)$ & $22.7(3.7)$ \\
\hline Subgenual—right & $11.7(2.1)$ & $11.5(2.4)$ \\
\hline Subgenual-left & $10.2(1.8)$ & $9.8(2.0)$ \\
\hline Sensorimotor-right & $30.6(2.5)$ & $30.4(4.0)$ \\
\hline Sensorimotor-left & $28.8(2.5)$ & $28.6(3.4)$ \\
\hline Midtemporal-right & $16.3(1.8)$ & $16.2(1.9)$ \\
\hline Midtemporal-left & $15.9(2.0)$ & $15.5(1.8)$ \\
\hline Parieto-occipital-right & $67.3(6.7)$ & $68.5(8.5)$ \\
\hline Parieto-occipital-left & $67.9(8.1)$ & $67.2(8.1)$ \\
\hline Inferior occipital and cerebellum-right & $38.3(6.0)$ & $39.0(5.2)$ \\
\hline Inferior occipital and cerebellum—left & $32.9(11.7)$ & $29.1(15.9)$ \\
\hline
\end{tabular}

\section{RESULTS}

A total of 48 women met eligibility criteria, of whom 45 were randomized by the end of the study period (Fig. 2). Twenty-two women (49\%) were allocated to the intervention and 23 women $(51 \%)$ to the control group. Together, these 45 women had a total of 52 infants (including 7 pairs of twins). There were no instances of treatment discontinuation. Only 48 of these infants had MRI scans conducted:- postrandomization, one child per group died before term-equivalent age, and the families of two children (one per group) were unable to attend scans. Further, in 2 of the 48 MRI scans conducted (again, one infant per group) postacquisition analysis was not possible due to movement artifact. Thus, we present baseline characteristics from the 45 women and 52 infants enrolled in this study, but short-term medical and MRI results were from only the 46 infants with postacquisition MRI data.

Baseline comparability of treatment groups. Baseline characteristics were similar across the groups, both for the 45 women and the 52 infants enrolled in the study (Table 1). A majority of women were English speaking and had completed high school.

MRI results. Scans from 46 children (23 per group) were suitable for postacquisition analysis. Comparison of absolute volumes of cerebral tissue types revealed no difference between the intervention and control groups $(p>0.28$ for each tissue type, Table 2, Fig. $3 A$ ). There was no evidence for any betweengroup difference in the regional parcellated volumes (overall $p=$ 0.42 , Table 2, Fig. $3 B$ ).

Of the 46 infants with data of brain MRI, suitable diffusion data were acquired in only 27 cases, as this was the final sequence performed and 21 infants were not able to remain still for a sufficiently long period to allow satisfactory data 

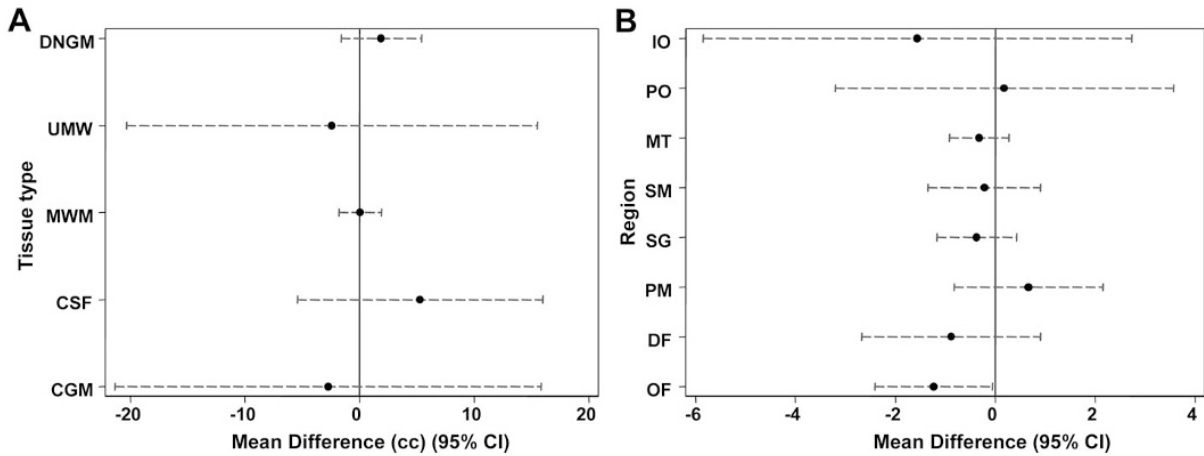

Figure 3. Differences in tissue volumes and regional brain volumes (intervention minus control). Mean differences in volume (mL) between the intervention and control group by type of tissue are plotted with $95 \%$ confidence intervals. The vertical reference line indicates zero difference. $A$. Cerebral tissue volumes: results are from separate univariable models for each tissue type. $B$. Regional brain volumes: estimates are from a single model combining data from all regions with a region-specific estimate of the difference across both hemispheres, using random effects to allow for correlations of observations within individuals. IO, inferior occipital; PO, parieto-occipital; MT, midtemporal; SM, sensorimotor; SG, subgenual; PM, premotor; DF, dorsal frontal; OF, orbitofrontal.
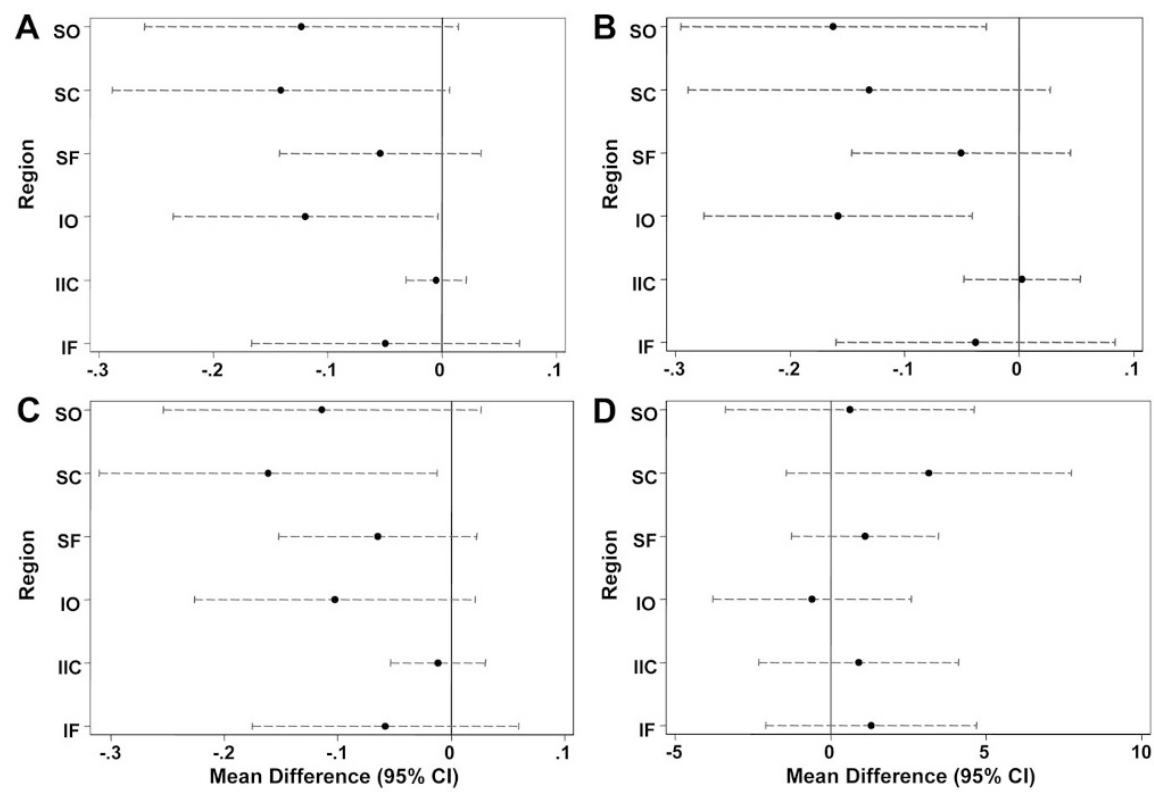

Figure 4. Mean difference in diffusion values (intervention minus control). Mean differences between the intervention and control group are plotted for each DTI parameter plotted along with $95 \%$ confidence intervals. The vertical reference line indicates zero difference. Estimates from a single model combining data from all regions with a region-specific estimate of the difference, combining the results across both hemispheres, using random effects to allow for the correlation of observations within individuals. A. ADC. B. Axial diffusivity. C. Radial diffusivity. D. FA. SO, superior occipital; SC, superior central; SF, superior frontal; IO, inferior occipital; IIC, inferior internal capsule; IF, inferior frontal.

Table 3. Mean $(S D)$ diffusion parameters by treatment group $(\mathrm{n}=15$ in the control group and $\mathrm{n}=12$ in the intervention group)

\begin{tabular}{|c|c|c|c|c|c|c|c|c|}
\hline \multirow[b]{2}{*}{ Region } & \multicolumn{2}{|c|}{$\mathrm{ADC}$} & \multicolumn{2}{|c|}{ Axial diffusivity } & \multicolumn{2}{|c|}{ Radial diffusivity } & \multicolumn{2}{|c|}{ FA } \\
\hline & Control & Intervention & Control & Intervention & Control & Intervention & Control & Intervention \\
\hline \multicolumn{9}{|l|}{ Lower slice } \\
\hline Frontal—right & $1.75(0.17)$ & $1.69(0.16)$ & $2.03(0.18)$ & $1.99(0.18)$ & $1.61(0.18)$ & $1.53(0.16)$ & $14.5(6.3)$ & $17.1(3.9)$ \\
\hline Frontal-left & $1.69(0.16)$ & $1.66(0.13)$ & $1.97(0.16)$ & $1.93(0.13)$ & $1.55(0.17)$ & $1.52(0.13)$ & $16.3(5.5)$ & $16.3(2.7)$ \\
\hline Internal capsule-right & $1.08(0.04)$ & $1.07(0.03)$ & $1.82(0.07)$ & $1.80(0.05)$ & $0.71(0.06)$ & $0.71(0.04)$ & $53.7(4.7)$ & $53.6(3.0)$ \\
\hline Sensorimotor-left & $1.09(0.05)$ & $1.09(0.04)$ & $1.78(0.10)$ & $1.81(0.07)$ & $0.74(0.07)$ & $0.73(0.06)$ & $50.6(5.9)$ & $52.5(4.7)$ \\
\hline Occipital—right & $1.70(0.15)$ & $1.60(0.18)$ & $2.01(0.13)$ & $1.85(0.17)$ & $1.55(0.17)$ & $1.48(0.19)$ & $17.6(5.9)$ & $16.0(4.0)$ \\
\hline Occipital-left & $1.73(0.14)$ & $1.59(0.19)$ & $2.03(0.13)$ & $1.87(0.24)$ & $1.59(0.15)$ & $1.46(0.18)$ & $16.6(5.4)$ & $17.0(3.7)$ \\
\hline \multicolumn{9}{|l|}{ Upper slice } \\
\hline Frontal-right & $1.66(0.09)$ & $1.62(0.16)$ & $1.93(0.10)$ & $1.89(0.18)$ & $1.53(0.10)$ & $1.48(0.16)$ & $16.2(2.8)$ & $17.1(4.0)$ \\
\hline Frontal-left & $1.63(0.12)$ & $1.57(0.12)$ & $1.93(0.16)$ & $1.86(0.10)$ & $1.50(0.12)$ & $1.43(0.13)$ & $17.8(3.6)$ & $19.1(4.7)$ \\
\hline Central-right & $1.52(0.21)$ & $1.41(0.14)$ & $1.80(0.23)$ & $1.70(0.16)$ & $1.38(0.21)$ & $1.26(0.15)$ & $18.2(6.7)$ & $20.1(5.9)$ \\
\hline Central-left & $1.59(0.25)$ & $1.41(0.18)$ & $1.88(0.28)$ & $1.73(0.17)$ & $1.46(0.23)$ & $1.26(0.21)$ & $17.2(6.7)$ & $21.6(7.8)$ \\
\hline Posterior-right & $1.67(0.19)$ & $1.54(0.15)$ & $1.98(0.18)$ & $1.80(0.14)$ & $1.53(0.19)$ & $1.42(0.15)$ & $17.8(6.4)$ & $16.6(4.3)$ \\
\hline Posterior-left & $1.67(0.24)$ & $1.56(0.15)$ & $1.99(0.24)$ & $1.85(0.16)$ & $1.52(0.24)$ & $1.41(0.15)$ & $16.8(7.0)$ & $19.2(4.7)$ \\
\hline
\end{tabular}

acquisition. Further, one child in the control group had aberrant DTI values lying several standard deviations from the mean. Analyses including and excluding this outlier produced similar results and conclusions were unchanged. For completeness, we present results including this individual's data.
ADCs were reduced in infants in the intervention group compared with controls for all regions (overall $p=0.0006$ ), with some suggestion that the effect of the intervention varied by region ( $p=0.10)$ but not by side ( $p=0.94$ : Fig. 4A, Table 3). 
Table 4. Key perinatal and medical characteristics of the 46 children with postacquisition MRI data

\begin{tabular}{lcc}
\hline & Control group $(n=23)$ & Intervention group $(n=23)$ \\
\hline Intraventricular hemorrhage (grade) & 3 (one with grade 2 and two with grade 3) & 3 (one infant each with grades 2,3, and 4) \\
Mean days in hospital (SD) & $88.6(27.8)$ & $82.3(24.4)$ \\
Mean postmenstrual age (weeks) at discharge (SD) & $40.4(2.8)$ & $39.5(2.1)$ \\
Sepsis in NICU, $n(\%)$ & $11(42)$ & $6(26)$ \\
Patent ductus arteriosus, $n(\%)$ & $17(74)$ & $16(70)$ \\
Indomethacin administered, $n(\%)$ & $12(52)$ & $13(57)$ \\
Median hours of ventilation & & \\
CPAP (IQR) & $456(72.0-688.0)$ & $612(97.5-1008.0)$ \\
IPPV (IQR) & $144(11.0-528.0)$ & $120(6.0-354.0)$ \\
Hours on $\mathrm{O}_{2}$ (IQR) & $216(7.0-1647.0)$ & $146(1.5-1008.0)$ \\
Still on $\mathrm{O}_{2}$ at discharge, $n(\%)$ & $5(22)$ & $6(26)$ \\
Mean postmenstrual age at MRI (SD) & $42.3(1.8)$ & $41.5(2.1)$ \\
Mean body weight in grams at MRI (SD) & $3209.3(504.8)$ & $3063.7(643.3)$ \\
\hline
\end{tabular}

CPAP, continuous positive airway pressure; IPPV, intermittent positive pressure ventilation; IQR, interquartile range.

The strongest effect appeared to be in the superior regions (Fig. 4A). This pattern was reflected in the axial and radial diffusivity (Fig. $4 B$ and $C$ ) with reduced diffusivity in children in the intervention group (overall $p=0.0003$ and 0.0007 for axial and radial diffusivity, respectively), and some suggestion that this effect varied by region, particularly for axial diffusivity (treatment-by-region interaction $p=0.05$ and 0.23 , respectively). These results were unchanged when adjusted for postmenstrual age at the time of MRI (not presented). Finally, there was some suggestion of an intervention effect on FA (overall $p=0.14$ ) but no evidence that this varied by region ( $p=0.86$; Table 3, Fig. 4A).

Perinatal medical variables. Table 4 shows the profile of the two groups in terms of these secondary outcomes. Although on average intervention group infants spent fewer days in hospital, fewer hours on oxygen, had fewer instances of sepsis, and were discharged at an earlier postmenstrual age (mean difference $6.3 \mathrm{~d}$ ) than control group infants, there were no statistically significant effects of treatment $(p>0.1$ in every case).

Frequencies of maternal care-giving behaviours. Between-group differences on three indicators of maternal caregiving were assessed by Mann-Whitney $U$ tests. Mothers in both groups were present with their hospitalized infants on the majority of days. Mothers in the intervention had visits with their infants on a median of $7 \mathrm{~d} /$ wk compared with $6.8 \mathrm{~d}$ in the control group $(p=0.08)$. Similarly, the weekly number of infant feeds administered by mothers had median values of 5.8 and 4.5 in the intervention and control groups, respectively, $(p=0.09)$. Periods of kangaroo care differed significantly between the groups (median of 1.7 kangaroo care periods per week in the intervention versus a median of 0 in the control group, $p=0.003$ ).

\section{DISCUSSION}

This is one of the few published trials of NICU-based early interventions for preterm children using MRI to assess the effects on early brain development. The fact that this study centers on parental sensitivity training in a population of preterm infants makes it unique.

The study by Als et al. (11) was the first to document evidence that environmental interventions (NIDCAP care) in preterm infants could be associated with altered cerebral structure and function. Although this does include parents, NIDCAP care focuses primarily on bedside-nurse input. MITP-type interventions also aim to reduce environmental stress but focus centrally on training the parent as the primary therapist to observe signs of stress and coping in their hospitalized infant and to interact in ways that ameliorate stress. A possible mechanism of MITP-type intervention is that infants can more consistently receive stress-sensitive care from a single individual (the mother). Potential advantages of MITPtype intervention are its relatively low cost, and the focal involvement of parents as the main change agents so that intervention can potentially continue postdischarge.

Our diffusion data suggest that our MITP-type intervention was associated with improved white matter microstructure reflected by lower ADC values. Diffusion measures in preterm infants, in particular ADC, have been shown to be altered at term-equivalent age and to be predictive of later neurodevelopmental outcomes (25). Preterm infants at term equivalent often display alterations in diffusion measures, with elevation in $\mathrm{ADC}$ and reduction in anisotropy relative to term control infants (26). Although no data on the neuropathological correlates for these alterations in white matter diffusion measures exist, it is plausible that they result from either delay in development and/or injury with loss of oligodendroglia and/or axonal components of the cerebral white matter. The white matter is known to be particularly vulnerable to injury in the preterm infant and often accompanied by a secondary cascade of effects on subsequent brain development (27). In contrast, we observed no statistically significant differences in total or regional brain volumes in our study.

There are several limitations to our study. The number of infants with MRI scans was small $(n=46)$. In addition, due to our broader inclusion criteria, our cohort was more heterogeneous than the sample $(n=30)$ studied by Als et al. (11), which may have introduced more variance from prenatal variables. However, this is potentially also a strength in outcomes that can be more confidently generalized to all preterm infants. In addition, our methods for the tissue segmentation and regional parcellation were semi-automated while diffusion analyses involved manual placement of ROIs. Despite improvements in acquisition time and motion correction, diffusion imaging remains a chal- 
lenging MR sequence in nonsedated infants. Recently, we have further optimized methods for stabilizing infants and reducing motion (28), which could help improve data acquisition in future work. Despite these limitations, these data remain consistent with those of Als et al. (11) and support the use of MRI methods as biomarkers for brain development.

We speculate that stress exposure in the immature brain has an adverse effect on cerebral injury and/or development such that reduction in stress via MITP-type intervention enhances cerebral white matter development as measured by ADC and anisotropy. This is supported by recent experimental data in the immature brain that has emphasized the capacity of mild chronic stress to worsen brain injury (29). In addition, stress exposure increases hypothalamic-pituitary-adrenal (HPA) hormonal reactions resulting in altered cerebral white matter development (30). It has been noted that sensitive maternal involvement has a mitigating effect on such neural changes (31). Finally, the positive impact of NIDCAP care interventions aimed at reducing infant stress on cerebral MRI and neurophysiological measures reported by Als et al. (11) is consistent with reduced cerebral white matter injury and/or improved cerebral development.

Although nonsignificant, perinatal, and medical outcomes appeared to be in line with a more general improvement in outcome for intervention children in terms of days on oxygen and length of stay reported in previous work. Als (16) found that preterm infants who had NIDCAP care required fewer ventilated days and days on continuous positive air pressure, achieved full oral feeds sooner and had a shorter, cheaper hospital stay. These patterns also appear to be consistent with the data presented by Westrup et al. (32).

Details of possible physiologic mechanisms of the intervention's effect are difficult to speculate on. Our study included no intermediate biomarkers that might indicate the nature of such processes, and this could be an important feature of future work.

In conclusion, parent-centered sensitivity training appears to be a promising approach to early intervention in the NICU with early short-term benefits for brain development that could contribute to later advantages in cognitive function reported by others (17). The findings of this study concur with recent evidence that the quality of early experience influences cerebral development. The results provide a platform for consideration of larger randomized trials of MITP-type interventions in preterm infants, and emphasize the utility of MRI as an early biomarker for cerebral injury and development.

Acknowledgments. We thank the families who took part in this study.

\section{REFERENCES}

1. Saigal S, Doyle LW 2008 An overview of mortality and sequelae of preterm birth from infancy to adulthood. Lancet 371:261-269

2. Inder TE, Warfield SK, Hong W, Huppi PS, Volpe JJ 2005 Abnormal cerebral structure is present at term in premature infants. Pediatrics 115:286-294

3. Thompson DK, Wood SJ, Doyle LW, Warfield SK, Lodygensky GA, Anderson PJ, Egan GF, Inder TE 2008 Neonatal hippocampal volumes: prematurity, perinatal predictors, and 2-year outcome. Ann Neurol 63:642-651
4. Woodward LJ, Anderson PJ, Austin NC, Howard K, Inder TE 2006 Neonatal MRI to predict neurodevelopmental outcome in preterm infants. N Engl J Med 355:685-694

5. Beauchamp MH, Thompson DK, Howard K, Doyle LW, Egan GF, Inder TE, Anderson PJ 2008 Preterm infant hippocampal volumes correlate with later working memory deficits. Brain 131:2986-2994

6. Nosarti C, Rushe TM, Woodruff PW, Stewart AL, Rifkin L, Murray RM 2004 Corpus callosum size and very preterm birth: relationship to neuropsychological outcome. Brain 127:2080-2089

7. Isaacs EB, Lucas A, Chong WK, Wood SJ, Johnson CL, Marshall C, VarghaKhadem F, Gadian DG 2000 Hippocampal volume and everyday memory in children of very low birth weight. Pediatr Res 47:713-720

8. Peterson BS, Vohr B, Staib LH, Cannistraci CJ, Dolberg A, Schneider KC, Katz KH, Westerveld M, Sparrow S, Anderson AW, Duncan CC, Makuch RW, Gore JC, Ment LR 2000 Regional brain volume abnormalities and long-term cognitive outcome in preterm infants. JAMA 284:1939-1947

9. Vohr BR, Wright LL, Dusick AM, Mele L, Verter J, Steichen JJ, Simon NP, Wilson DC, Broyles S, Bauer CR, Delaney-Black V, Yolton KA, Fleisher BE, Papile LA, Kaplan MD 2000 Neurodevelopmental and functional outcomes of extremely low birth weight infants in the National Institute of Child Health and Human Development Neonatal Research Network 1993-1994. Pediatrics 105:1216-1226

10. Rickards AL, Kitchen WH, Doyle LW, Ford GW, Kelly EA, Callanan C 1993 Cognition, school performance and behavior in very low birth weight and normal birth weight children at 8 years of age: a longitudinal study. J Dev Behav Pediatr 14:363-368

11. Als H, Duffy FH, McAnulty GB, Rivkin MJ, Vajapeyam S, Mulkern RV, Warfield SK, Huppi PS, Butler SC, Conneman N, Fischer C, Eichenwald EC 2004 Early experience alters brain function and structure. Pediatrics 113:846-857

12. Newnham CA, Milgrom J, Skouteris H 2009 Effectiveness of a modified MotherInfant Transaction Program on outcomes for preterm infants from 3 to 24 months of age. Infant Behav Dev 32:17-26

13. Vázquez DM, Lopez JF, Van Hoers H, Watson SJ, Levine S 2000 Maternal deprivation regulates serotonin $1 \mathrm{~A} \& 2 \mathrm{~A}$ receptors in the infant rat. Brain Res 855:76-82

14. Carlson M, Earls F 2000 Social ecology and the development of stress regulation. In: Bergman LR, Cairns RB, Nilsson, Nystedt L (eds) Developmental Science and the Holistic Approach. Lawrence Erlbaum Associates, Mahwah, NJ, pp 229-248

15. Als H 1990 Newborn Individualized Developmental Care and Assessment Program (NIDCAP) Training Guide. Children's Hospital, Boston, MA

16. Als H 1999 Reading the premature infant. In: Goldson (ed) Nurturing the Premature Infant: Developmental Interventions in the Neonatal Intensive Care Nursery. Oxford University Press, New York, NY, pp 18-85

17. Rauh VA, Nurcombe B, Achenbach T, Howell C 1990 The Mother-infant transaction program. Clin Perinatol 17:31-45

18. Achenbach TM, Howell CT, Aoki MF 1993 Nine-year outcome of the Vermont Intervention Program for low-birthweight infants. Pediatrics 91:45-55

19. Inder TE, Anderson NJ, Spencer C, Wells S, Volpe JJ 2003 White matter injury in the premature infant: a comparison between serial cranial sonographic and MR findings at term. AJNR Am J Neuroradiol 24:805-809

20. Warfield S 1996 Fast k-NN classification for multichannel image data. Pattern Recognit Lett 17:713-721

21. Warfield SK, Kaus M, Jolesz FA, Kilinis R 2000 Adaptive, template moderated, spatially varying statistical classification. Med Image Anal 4:43-55

22. Stejskal EO, Tanner JE 1965 Spin diffusion measurements: spin echoes in the presence of a time-dependent field gradient. J Chem Phys 42:288-292

23. Moher D, Schulz KF, Altman DG 2001 The CONSORT statement: revised recommendations for improving the quality of reports of parallel-group randomised trials. Lancet 357:1191-1194

24. Altman DG 1998 Adjustment for covariate imbalance. In: Armitage P, Colton T (eds) Encyclopedia of Biostatistics. John Wiley, Chichester, England, pp 1000-1005

25. Krishnan ML, Dyet LE, Boardman JP, Kapellou O, Allsop JM, Cowan F, Edwards AD, Rutherford MA, Counsell SJ 2007 Relationship between white matter apparent diffusion coefficients in preterm infants at term-equivalent age and developmental outcome at 2 years. Pediatrics 120:e604-e609

26. Rose SE, Hatzigeorgiou X, Strudwick MW, Durbridge G, Davies PS, Colditz PB 2008 Altered white matter diffusion anisotropy in normal and preterm infants at term-equivalent age. Magn Reson Med 60:761-767

27. Volpe JJ 2009 Brain injury in premature infants: a complex amalgam of destructive and developmental disturbances. Lancet Neurol 8:110-124

28. Mathur AM, Neil JJ, McKinstry RC, Inder TE 2008 Transport, monitoring and successful brain MR imaging in unsettled neonates. Pediatr Radiol 38:260-264

29. Rangon C-M, Fortes S, Lelièvre V, Leroux P, Plaisant F, Joubert C, Lanfumey L, Cohen-Salmon C, Gressens P 2007 Chronic mild stress during gestation worsens neonatal brain lesions in mice. J Neurosci 27:7532-7540

30. Kapoor A, Dunn E, Kostaki A, Andrews MH, Matthews SG 2006 Fetal programming of hypothalamo-pituitary-adrenal function: prenatal stress and glucocorticoids. J Physiol 572:31-44

31. Macri S, Chiarotti F, Wurbel H 2008 Maternal separation and maternal care act independently on the development of HPA responses in male rats. Behav Brain Res 191:227-234

32. Westrup B, Kleberg A, von Eichwald K, Stjernqvist K, Lagercrantz H 2000 A randomized controlled trial to evaluate the effects of the newborn individualized developmental care and assessment program in a Swedish setting. Pediatrics 105:66-72 